Original study

\title{
Primary meningeal sarcoma in children: a survey from the French Society of Pediatric Oncology (SFCE)
}

\author{
Abdullah Almasoud ${ }^{1,{ }^{*}}$, Emmanuelle Schmitt ${ }^{2}$, Jacques Grill ${ }^{3}$, Didier Frappaz ${ }^{4}$, Jean- \\ Claude Gentet $^{5}$, Sandrine Pall-Kondollf ${ }^{1}$, Marie-Amelyne Le Rouzic ${ }^{1}$, Julie Valduga ${ }^{1}$ \\ ${ }^{1}$ Department of Pediatric Hematology and Oncology, Children's University Hospital, Nancy, \\ France, ${ }^{2}$ Department of Pediatric Neuro-Radiology, Children's University Hospital, Nancy, France, \\ ${ }^{3}$ Department of Pediatric Hematology and Oncology, Gustave Roussy Institute, Villejuif, France, \\ ${ }^{4}$ Department of Pediatric Hematology and Oncology, Institute of Pediatric Hematology and Oncology, \\ Lyon, France, ${ }^{5}$ Department of Pediatric Hematology and Oncology, La Timone Hospital, Marseille, \\ France
}

\begin{abstract}
Objectives: To describe the outcome of primary meningeal sarcoma (PMS) in a pediatric population. Methods: We conducted a retrospective study on patients harbouring a PMS based on the French registry of paediatric tumours. Questionnaires were sent to all French paediatric oncologists. Data on patient characteristics and treatments were collected. Pathology and imaging were centrally reviewed. Results: Between August 1989 and May 2010, 12 patients from 6 French centres, aged 3 months to 14.5 years (mean: 3.3 years) were treated for a PMS. Mean follow-up was 12 years (range: 3 months to 24 years). Tumour locations were: frontal (3), parieto-occipital (2), parietal (1), temporal (1), occipital (1), thalamic (1), pontocerebellar angle (1), cerebellar tentorium (1), ambient cistern (1). No metastasis was observed. The firstline treatment was surgery in 10 cases, chemotherapy in 2 . Resection was total in 6 cases, partial in 6 . Central pathology review concluded to: high-grade undifferentiated sarcoma (8), chondrosarcoma (2), fibrosarcoma (1), myxoid desmoplastic tumour (1). Seven tumours were smaller than $5 \mathrm{~cm}$ in size. Eleven patients received 2 to 10 courses of chemotherapy (median: 5 ) but no response was observed. Four out of 6 patients for whom a total resection was performed were still alive at time of study, compared to 2 when only partial resection was possible. Four out of 6 patients who received radiotherapy are still alive, compared to 1 out of 4 patients without radiotherapy. The 5-year Event-Free Survival (EFS) and Overall Survival (OS) rates were 50\%. The median EFS in case of total resection was 39 months versus 16 months in case of partial resection. Neither correlation was found between tumour size and location or between age at diagnosis and EFS/OS. Conclusions: We report the first multicentre case series describing clinical, radiological presentation and outcome of PMS in children. In this short series of very rare tumours, age and tumour size did not seem to be prognostic factors. Total resection and radiotherapy seem to be essential. The role of chemotherapy remains unclear.
\end{abstract}

Keywords: : meningeal sarcoma; child, surgery; chemotherapy; radiotherapy; outcome

Received: February 2018; Accepted after review: May 2018; Published: June 2018.

${ }^{*}$ Corresponding author: Abdullah Almasoud, Department of Pediatric Hematology and Oncology, Children's University Hospital, 11 Allée du Morvan, 54511 Vandoeuvre, France.

Email: dr.a.almasoud@gmail.com

\section{Introduction}

Primary meningeal sarcomas are extremely rare in childhood. Meningeal tumours account for less than $2 \%$ of all intracranial tumours in children under the age of 15 , and less than $5 \%$ in adolescents $[1,2]$ but the incidence of PMS remains unknown. 
Reports on paediatric PMS are limited to case reports or very small single-institutional series precluding a proper assessment of clinical course and ideal treatment plan [2-12]. The main presenting symptoms are related to tumour location, size and aggressiveness. They are characterized by short clinical history, rapid deterioration and high recurrence after surgical resection. The mean 1-year survival rate is around $50 \%$ [2-11]. There is no guideline for treatment but total surgical resection and radiotherapy play an important role while the effectiveness of chemotherapy remains to be demonstrated. This study reports the largest series of paediatric PMS and aims at analysing their clinical behaviour, radiological and pathological characteristics in an attempt to recommend treatment options for PMS in comparison to other meningeal tumours.

\section{Methods}

This study is a retrospective multicentre study. Patients who were diagnosed with PMS between August 1989 and May 2010 within the French Society of Pediatric Oncology (SFCE) centres have been recruited from the French oncology registry. All clinical and therapeutic data have been collected from standardized questionnaires sent to the SFCE paediatric oncologists. Complete staging was reviewed including spinal MRI and cytological analysis of cerebrospinal fluid (CSF). In addition, central review of pathological diagnosis and radiological imaging was performed. Survival probability was estimated by the Kaplan-Meyer method. OS was defined as the time between the date of diagnosis and the date of the latest follow-up visit or date of death of any cause. Progression-free survival (PFS) was defined as the time between the diagnosis and the earliest evidence of first progression, relapse, occurrence of secondary malignancy or death of any cause.

\section{Results}

Clinical characteristics
Twelve patients (6 males and 6 females) were included from six referral French centres during the period of observation. The clinical characteristics of study patients are shown in Table 1. Median age at diagnosis was 6 years (range: 3 months to 14.5 years). None of the patients had evidence of neurofibromatosis type 2. Median duration of symptoms prior to diagnosis was 1 month (range: 10 days to 10 weeks). Median follow-up was 12 years (range: 3 months to 24 years). The main presentation included clinical features of raised intracranial pressure (7 patients) or neurological deficits (4 patients). One patient presented to the emergency room and was admitted for traumatic brain injury. No case of seizure was observed.

\section{Radiological characteristics}

In all cases, the MRI images showed the tumour either as a homogeneous or heterogeneous circular non-capsulated mass. Tumour locations were: frontal (3), parietooccipital (2), parietal (1), temporal (1), occipital (1), thalamic (1), pontocerebellar angle (1), cerebellar tentorium (1), ambient cistern (1). Calcifications were observed in 1 case and so was an isolated cystic lesion. Tumours smaller than $5 \mathrm{~cm}$ in size were found in 7 cases while tumours larger than $5 \mathrm{~cm}$ in size were found in 5 cases. MRI showed a marked contrast enhancement in all cases. Cystic structures were observed in most of the tumours. No metastasis was observed. (Figures 1-4).

\section{Pathology \\ After central pathology review, histopathological diagnoses included high- grade undifferentiated sarcoma (8), chondrosarcoma (2), fibrosarcoma (1), and myxoid desmoplastic tumour (1). On immunohistochemistry examination, vimentin intermediate filaments were mostly detected and there were no specific biological entities of PMS.}

\section{Treatments}

The first-line treatment was surgery in 10 cases, chemotherapy in 2 . Resection was total in 6 cases, partial in 4. A biopsy was performed in 2 patients. 
Eleven patients received 2 to 10 courses of chemotherapy (median: 5). It was administered as neoadjuvant treatment in 2 cases, as adjuvant therapy in 8 cases and as salvage therapy in 1 case. Most of the time, the regimen included vincristine, doxorubicine, etoposide, carboplatine and ifosfamide. No response was observed among 7 patients with measurable tumour volume.

Nine patients received radiotherapy on the tumour bed, as adjuvant therapy in 8 cases and as salvage therapy in 1 case. The radiation dose ranged from 50.4 to $56.2 \mathrm{~Gy}$ (median: $54 \mathrm{~Gy}$ ).

\section{Outcome}

At last follow-up, six patients were alive, five in first remission and one in second remission. One patient relapsed with lung metastasis. One patient died of secondary cancer (acute lymphoblastic leukemia).

Four out of 6 patients for whom a total resection was performed were still alive at time of study, compared to 2 when only partial resection was possible.

The median EFS in case of total resection was 39 months versus 16 months in case of partial resection. Five out of 9 patients under the age of 10 years were alive with a median follow-up of 118 months (range: 102 to 168 months), while 1 out 3 patients above the age of 10 years was alive. Four out of 7 patients with a tumour size smaller than $5 \mathrm{~cm}$ were alive versus 3 out of 5 patients with a tumour size larger than $5 \mathrm{~cm}$. Four out of 6 patients who received radiotherapy were alive versus 1 out of 4 without radiotherapy. The 5-year EFS and OS rates were $50 \%$.

Table 1. Main clinical, histopathological and treatment characteristics of patients

\begin{tabular}{|c|c|c|c|c|c|c|c|c|}
\hline Patient & $\begin{array}{c}\text { Age } \\
\text { (years) }\end{array}$ & $\begin{array}{l}\text { Tumour } \\
\text { Location }\end{array}$ & $\begin{array}{l}\text { Tumour size } \\
\text { (cm) }\end{array}$ & Histology & Resection & $\begin{array}{l}\text { Chemo- } \\
\text { therapy }\end{array}$ & $\begin{array}{l}\text { Radio- } \\
\text { therapy }\end{array}$ & $\begin{array}{l}\text { OS status } \\
\text { (months) }\end{array}$ \\
\hline 1 & 8 & Frontal & $>5$ & $\begin{array}{c}\mathrm{HG} \\
\text { sarcoma }\end{array}$ & Total & + & + & $\begin{array}{c}30 \\
\text { DOD }\end{array}$ \\
\hline 2 & 7 & Occipital & $>5$ & $\begin{array}{c}\text { Fibro- } \\
\text { sarcoma }\end{array}$ & Partial & + & - & $\begin{array}{c}12 \\
\text { DOD }\end{array}$ \\
\hline 3 & 12 & Thalamic & $>5$ & $\begin{array}{l}\text { Chondro- } \\
\text { sarcoma }\end{array}$ & Partial & + & + & $\begin{array}{c}27 \\
\text { DO ALL }\end{array}$ \\
\hline 4 & 2 & Frontal & $<5$ & $\begin{array}{c}\mathrm{HG} \\
\text { sarcoma }\end{array}$ & Partial & + & - & $\begin{array}{c}2 \\
\text { DOD }\end{array}$ \\
\hline 5 & 0.3 & $\begin{array}{l}\text { Ponto- } \\
\text { cerebellar }\end{array}$ & $<5$ & $\begin{array}{c}\mathrm{HG} \\
\text { sarcoma }\end{array}$ & Partial & + & - & $\begin{array}{c}7 \\
\text { DOD }\end{array}$ \\
\hline 6 & 14.5 & Occipital & $<5$ & $\begin{array}{c}\mathrm{HG} \\
\text { sarcoma }\end{array}$ & Total & + & + & $\begin{array}{c}36 \\
\text { DOD }\end{array}$ \\
\hline 7 & 3 & Ambient Cistern & $>5$ & $\begin{array}{c}\mathrm{HG} \\
\text { sarcoma }\end{array}$ & Partial & + & + & $\begin{array}{l}168 \\
\text { CR1 }\end{array}$ \\
\hline 8 & 3 & Temporal & $>5$ & $\begin{array}{c}\mathrm{HG} \\
\text { sarcoma }\end{array}$ & Partial & + & + & $\begin{array}{l}128 \\
\text { CR1 }\end{array}$ \\
\hline 9 & 5 & Frontal & $<5$ & $\begin{array}{l}\text { Chondro- } \\
\text { sarcoma }\end{array}$ & Total & + & + & $\begin{array}{l}108 \\
\text { CR1 }\end{array}$ \\
\hline 10 & 2 & $\begin{array}{l}\text { Cerebellar } \\
\text { tentorium }\end{array}$ & $<5$ & $\begin{array}{c}\text { Desmo-plastic } \\
\text { tumour }\end{array}$ & Total & + & + & $\begin{array}{l}102 \\
\text { CR1 }\end{array}$ \\
\hline 11 & 4 & $\begin{array}{l}\text { Parieto- } \\
\text { occipital }\end{array}$ & $<5$ & $\begin{array}{c}\mathrm{HG} \\
\text { sarcoma }\end{array}$ & Total & + & + & $\begin{array}{l}108 \\
\text { CR2 }\end{array}$ \\
\hline 12 & 10.5 & $\begin{array}{l}\text { Parieto- } \\
\text { occipital }\end{array}$ & $<5$ & $\begin{array}{c}\mathrm{HG} \\
\text { sarcoma }\end{array}$ & Total & + & - & $\begin{array}{l}168 \\
\text { CR1 }\end{array}$ \\
\hline
\end{tabular}

HG: High Grade, OS: Overall Survival, DOD: Dead Of Disease, CR1: First Complete Remission, CR2: Second Complete Remission, ALL: Acute Lymphoblastic Leukaemia 


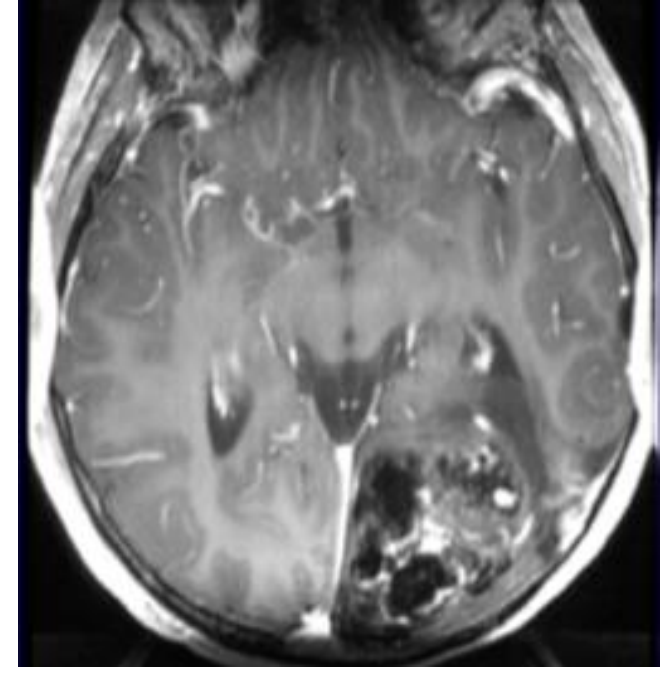

Fig. 1. High grade sarcoma: Axial post-contrast T1W image. Heterogeneous left occipital tumor with irregular and nodular enhancement

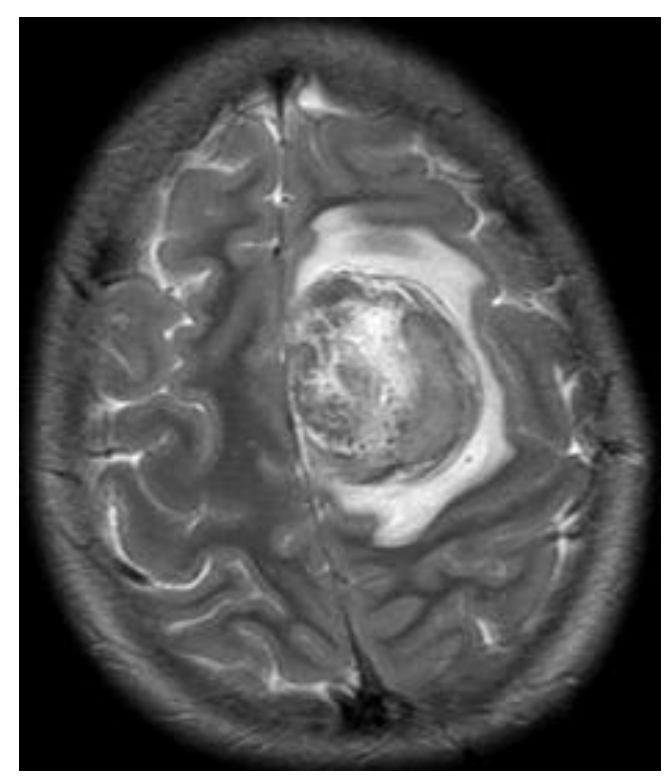

Fig.3. High grade sarcoma: Axial T2W image. Heterogeneous left frontal lesion with surrounding edema

\section{Discussion}

Our series of 12 patients from six French referral centres over 20 years represents the largest series reported in the literature. It confirms that meningeal sarcomas are very rare and aggressive entities without any predictive prognostic factors. PMS are particularly rare among meningeal tumours. They represent 1 to $4 \%$ of paediatric brain tumours, less than $20 \%$ of which are classified

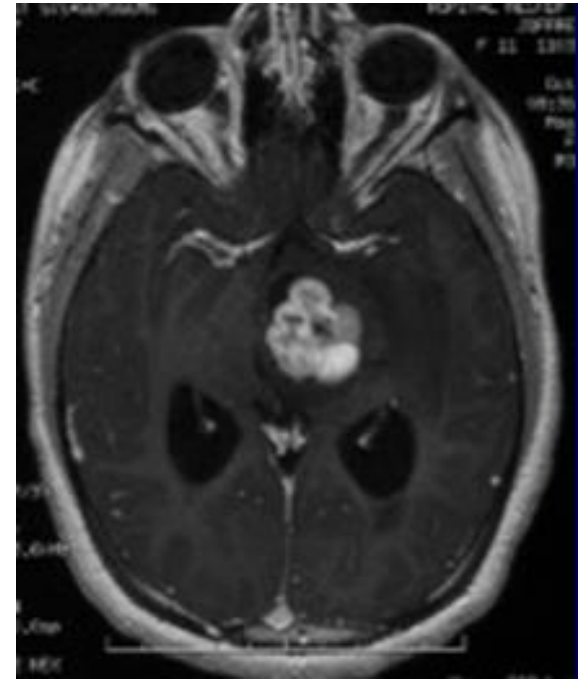

Fig. 2. Chondrosarcoma: Axial post-contrast T1W image. Left deep lesion, well delimited with high enhancement and microcysts

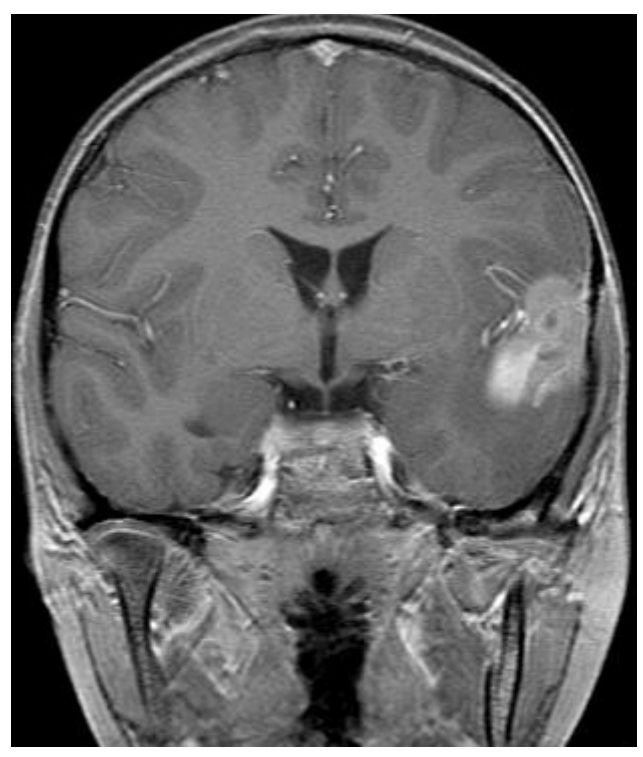

Fig.4. High grade sarcoma: Coronal post-contrast T1W image. Fronto-temporal left lesion moderately enhanced with peripheral blurring

as malignant [13], and very few of which are represented by PMS $[2,7,12]$. The incidence of primary intracranial soft tissue sarcomas remains unknown, estimated between 0.1 and $4 \%$ of intracranial paediatric tumours [15]. Among them, meningeal tumours are also very rare $[2,14,15]$. There are only few cases reported in the literature which described the clinical presentation, the histopathological findings and the treatment experience. Indeed, we found only 31 reported cases, only 13 of 
which are relatively well described in the literature published from 1992 to 2016 [8-11]. Moreover, the definition of meningeal sarcoma was a subject of discussion over the past decades. According to Haddad et al., the term "meningeal sarcoma" designates superficial intracranial sarcomas involving the leptomeninges [16]. Rubinstein et al. suggested that primary meningeal sarcoma is a tumour originating from the leptomeninges with infiltration of brain tissue and usually displaying immunoreactivity only for vimentin (and no immunoreactivity found in other possible tumours such as carcinoma, lymphoma, glioma or PNET) [17]. They can differentiate and present as fibrosarcoma, chondrosarcoma, rhabdomyosarcoma, leiomyosarcoma, osteosarcoma, malignant fibrous histiocytoma or angiosarcoma [16]. Four different pathologies were observed in our series of 12 patients and undifferentiated sarcoma was mostly represented (8/12). The same proportion (13/18) was reported in the literature [2-11].

Our results confirm that there is neither specific tumour location nor prognostic value for age or sex [2-11]. Interestingly, the median age reported in the largest series is 11 yearsold for meningeal tumours and 7 years-old for intracranial sarcomas while it is 2 years-old for PMS in the literature and 3 years-old in our series $[2,7,8,12,14,15]$. PMS patients seem to be younger, increasing the negative longterm effects of cranial radiotherapy which is, however, necessary. Symptoms at presentation (such as seizures, weakness, giddiness and visual disturbances) were not specific but rather related to tumour location and increased intracranial pressure. There are no pathognomonic radiological features specific for PMS. Our results showed that the majority of patients with evidence of a spaceoccupying brain lesion on the initial MRI present a non-capsulated homogeneous or heterogeneous mass.

Different series of paediatric meningioma reported an association between childhood meningioma and neurofibromatosis [18]. No patient had evidence of neurofibromatosis in our series and we did not find any case of neurofibromatosis in paediatric PMS case reports.
Since the first reported case in the literature, treatment modalities of PMS remain a subject of controversy because of its rarity and erratic follow-up. The first step is to make the correct diagnosis through open brain biopsy or surgical resection for all suspicious brain masses with meningeal involvement. The gold standard treatment for intracranial meningioma is surgical resection with total excision, resulting in long-term complete remission [19]. However, while meningeal tumours are classified as non-malignant tumours in about $80 \%$ of the cases, PMS are highly malignant tumours and we found only one case of long-term survival ( 1 year) among patients treated with radical resection only [11]. Moreover, meningioma resection in childhood is a neurosurgical challenge because of their frequent large size, possible adherence to major intracranial vessels leading to potential massive intraoperative blood loss and need for transfusion, particularly in the case of PMS [13]. The role of chemotherapy and radiation therapy is not clear yet. Radiotherapy is generally reserved for children with residual lesions, recurrent meningiomas or malignant types of meningioma [18]. Tumour excision with adjuvant radiotherapy improves the recurrence-free survival rate in children with malignant meningiomas other than PMS [18]. Out of 5 PMS patients for whom the extent of surgical resection was reported, it was considered total in two cases and the patients were alive at 7 months and 16 years after the end of treatment combining chemotherapy and radiotherapy [2]. In case of partial resection, 2 patients died of disease ant 1 patient relapsed $[2,12]$. Resection was considered total in 6 out of our 12 patients, confirming the impact of total resection since only 1 out of 4 patients with partial resection (median EFS: 16 months) was alive versus 4 out of 6 with total resection (median EFS: 39 months). The role of radiotherapy also seems to be a prerequisite to achieve long-term disease control, as observed for intracranial sarcomas [14]. In our study, while all patients received chemotherapy, no patient survived who underwent partial resection without additional radiotherapy. Five out of 8 patients who received radiotherapy were alive versus 1 out 
of 4 without radiotherapy. However, the adverse effects of radiation therapy on such a young population (median age: 2 to 3 years) remain of significant concern. On the contrary, the role of chemotherapy is less clear. Benesch et al. reported that there is no specific combination of drugs, duration or schedule of treatment for children harbouring intracranial sarcomas but suggested to deliver chemotherapy before and after radiotherapy, once radical surgical resection is performed [14]. Maher et al. concluded that a 5 drugregimen (vincristine, doxorubicin, cyclophosphamide, etoposide and ifosfamide) an ICE regimen (ifosfamide, carboplatin, etoposide) are valid treatment approaches along with total surgical resection and radiation therapy in this population of patients [15]. PMS can metastasize outside the nervous system as observed in one of our cases who relapsed with lung metastases, implying that post-treatment surveillance recommendations have to be implemented based on the experience of other sarcoma locations.

In summary, to the best of our knowledge, this is the first multicentre paediatric series to report clinical, radiological presentation and outcome of PMS in children. The limitations of our study include its retrospective nature and the small number of cases. Patients presented

\section{References}

1. Dolecek TA, Propp JM, Stroup NE, et al. CBTRUS statistical report: primary brain and central nervous system tumors diagnosed in the United States in 2005-2009. Neuro Oncol 2012; 14:1-49.

2. Al-Gahtany $M$, Shroft $M$, Bouffet $E$, et al. Primary central nervous system sarcomas in children: clinical, radiological, and pathological features. Childs Nerv Syst 2003;19:808-817.

3. Hsü Yk. Primary intracranial sarcomas. Arch Neurol Psychiat 1940; 43:901-924.

4. Onofrio BM, Kernohan JW, Uihlein A. Primary meningeal sarcomatosis. A review of the literature and report of 12 cases. Cancer 1962; 15:1197-1208.

5. Budka H, Pilz P, Guseo A. Primary leptomeningeal sarcomatosis. Clinicopathological report of six cases. J Neurol 1975; 211:77-93. with miscellaneous tumour locations and histological features. All ages were represented. Due to the small number of patients and the long follow-up period, no definitive conclusion could be drawn. Age, tumour location and size did not seem to be prognostic factors. We confirm the importance of total surgical resection and additional radiotherapy, while the role of chemotherapy is difficult to assess. The 5-year EFS and OS rates are $50 \%$. Long-term neurocognitive and endocrine sequelae are frequent, partly due to radiotherapy.

We suggest the inclusion of this type of rare tumours in a specific multicentre prospective international study to gather more clinical information which would help defining recommendations to implement a standardized protocol of treatment.

\section{Abbreviations}

PMS: Primary Meningeal Sarcoma; EFS: EventFree Survival; OS: Overall Survival; PFS: Progression-Free Survival; ALL: Acute Lymphoblastic Leukemia

\section{Conflict of interests}

The authors have no conflict of interest to declare.
6. Kishikawa $T$, Numaguchi $Y$, Fukui $M$, et al. Primary intracranial sarcomas: radiological diagnosis with emphasis on arteriography. Neuroradiology 1981; 21:25-31.

7. Davidson GS, Hope JK. Meningeal tumors of childhood. Cancer 1989; 63:1205-1210.

8. Hope JK, Armstrong DA, Babyn PS, et al. Primary meningeal tumors in children: correlation of clinical and CT findings with histologic type and prognosis. $A m \mathrm{~J}$ Neuroradiol 1992; 13:1353-1364.

9. Büttner $A$, Pfluger $T$, Weis $S$. Primary meningeal sarcomas in two children. $J$ Neurooncol 2001; 52:181-188.

10. Cummings $M$, Chowdhry $V$, Shah $H$, et al. Recurrent meningeal sarcoma successfully treated with stereotactic radiosurgery. J Neurosurg Pediatr 2012; 10:434-438. 
11. Singla N, Kapoor A, Chatterjee D. Undifferentiated meningeal sarcoma of childhood presenting as hard mass adhered to major intracranial vessels. Childs Nerv Syst 2016; 32:771-773.

12. Paulus W, Slowik F, Jellinger K. Primary intracranial sarcomas: histopathological features of 19 cases. Histopathology 1991; 18:395-402.

13. Wang $X Q$, Jiang $C C$, Zhao $L$, et al. Clinical features and treatment of World Health Organization Grade II and III meningiomas in childhood: report of 23 cases. I Neurosurg Pediatrics 2012; 10:423-433.

14. Benesch $M$, von Bueren $A O$, Dantonello $T$, et al. Primary intracranial soft tissue sarcoma in children and adolescents: a cooperative analysis of the European CWS and HIT study groups. J Neurooncol 2013; 111:337-345.

15. Maher OM, Khatua S, Mukherjee D, et al. Primary intracranial soft tissue sarcomas in children, adolescents, and young adults: single institution experience and review of the literature. J Neurooncol 2016; 127:155-163.

16. Haddad GF, Al-Mefty $O$. Meningeal sarcoma. In: Kaye AH, Laws ERJ (eds). Brain tumors. An encyclopedic approach. Churchill Livingstone, Edinburgh; 1995. p 713-721.

17. Rubinstein L, Russell D. Pathology of Tumours of the Nervous System. 5th ed. London: Edward Arnold; 1989. p. 507-532.

18. K Ravindranath, MC Vasudevan, A Pande, et al. Management of pediatric intracranial meningiomas: an analysis of 31 cases and review of literature. Childs Nerv Syst 2013; 29:573-582.

19. Dziuk TW, Woo S, Butler EB, et al. Malignant meningioma: an indication for initial aggressive surgery and adjuvant radiotherapy. $J$ Neurooncol 1998; 37:177-188. 